\title{
Frequent mono-allelic loss associated with deficient PTEN expression in imatinib-resistant gastrointestinal stromal tumors
}

\author{
Anna Quattrone ${ }^{1}$, Agnieszka Wozniak ${ }^{2}$, Barbara Dewaele ${ }^{1}$, Giuseppe Floris ${ }^{2,3}$, \\ Vanessa Vanspauwen ${ }^{1}$, Thomas Van Looy ${ }^{2}$, Patrick Schöffski ${ }^{2}$, Piotr Rutkowski ${ }^{4}$, Raf Sciot ${ }^{3}$ \\ and Maria Debiec-Rychter ${ }^{1}$ \\ ${ }^{1}$ Department of Human Genetics, KU Leuven and University Hospitals Leuven, Leuven, Belgium; ${ }^{2}$ Laboratory \\ of Experimental Oncology, Department of General Medical Oncology, KU Leuven and University Hospitals \\ Leuven, Leuven, Belgium; ${ }^{3}$ Department of Pathology, KU Leuven and University Hospitals Leuven, Leuven, \\ Belgium and ${ }^{4}$ Department of Soft Tissue/Bone Sarcoma and Melanoma, M. Sklodowska-Curie Memorial \\ Cancer Center and Institute of Oncology, Warsaw, Poland
}

\begin{abstract}
Insufficiency of phosphatase and tensin homolog (PTEN) occurs in numerous tumor types and has been implicated as a resistance mechanism to receptor tyrosine kinase-targeted therapies in human cancer. In this study, we have performed a comprehensive molecular and immunohistochemical characterization of PTEN in 58 imatinib-naïve and 54 imatinib-treated gastrointestinal stromal tumors (GISTs). The findings were correlated with clinicopathological data. At the genomic level, PTEN was affected mainly by mono-allelic loss, which was significantly less frequent in imatinib-naïve vs imatinib-resistant tumors $(9 \%$ vs $39 \%, P<0.001)$. Neither $P$ TEN mutations nor PTEN promoter hyper-methylation were found. By immunohistochemistry, PTEN depletion was clearly related to GIST progression. Low PTEN protein expression was common $(50 \%)$ and often paralleled with total immunonegativity in imatinib-resistant tumors. The abnormal PTEN protein expression correlated with PTEN loss at the genomic level $(P=0.001)$. In addition, the effect of small interfering RNA (siRNA) PTEN knockdown on KIT signaling was examined in GIST-T1 and GIST430 cell lines, in the absence or presence of a dual PI3K/mTOR inhibitor NVP-BEZ235, alone or in combination with imatinib. In both cell lines, siRNA silencing of PTEN resulted in the substantial upregulation of PI3K-AKT and MAPK pathways. The MAPK hyperactivation was further potentiated by NVP-BEZ235 in the imatinib-sensitive GIST-T1 cells; yet, this effect was counteracted efficiently by combined treatment. In the imatinib-resistant GIST430 cells, neither NVP-BEZ235 alone or in combination with imatinib yielded sufficient inhibition of hyper-phosphorylated MAPK and downstream intermediate $\mathbf{S 6}$ protein. In conclusion, depleted PTEN expression associated with mono-allelic PTEN loss occurs frequently in imatinib-resistant GIST and might serve as a biomarker for stratifying patients for optimal treatment. In vitro, the PTEN insufficiency leads to hyperactivation of AKT and MAPK pathways in tumor cells. Novel therapies targeting multiple components of the integrated KIT receptor signaling pathways in imatinibresistant GIST warrant further studies.
\end{abstract}

Modern Pathology (2014) 27, 1510-1520; doi:10.1038/modpathol.2014.53; published online 18 April 2014

Keywords: gastrointestinal stromal tumor; imatinib mesylate; PTEN; resistance

The phosphatase and tensin homolog (PTEN) is a phosphatase able to convert membrane-associated

Correspondence: Professor/Dr M Debiec-Rychter, PhD, Department of Human Genetics, KU Leuven and University Hospitals Leuven, Herestraat 49, Leuven B-3000, Belgium.

E-mail: maria.debiec-rychter@med.kuleuven.be

Received 8 December 2013; revised 11 February 2014; accepted 26

February 2014; published online 18 April 2014 phosphatidylinositol 3,4,5-triphosphate back to phosphatidylinositol 4,5-bisphosphate; thereby it negatively regulates the signaling transduction of the PI3K/AKT pathway. ${ }^{1}$ After TP53, PTEN represents the second most frequently mutated tumorsuppressor gene in cancer. PTEN inactivation has a role in several human neoplasms, including glioblastoma, endometrial, prostate, colon, and breast carcinoma. In some of these tumors it has been 
demonstrated that PTEN deficiency is associated with advanced tumor stage and therapeutic resistance, especially to targeted therapies of receptor tyrosine kinases and their pathways. ${ }^{2}$ In breast cancer, PTEN loss confers resistance to the antiHER2 antibody trastuzumab. ${ }^{3}$ Similarly, it promotes resistance to EGFR tyrosine kinase inhibitors in glioblastoma, colon, and lung cancer. ${ }^{4-6}$

In gastrointestinal stromal tumors (GISTs), KIT and PDGFRA are fundamental therapeutic targets; however, the majority of GIST patients eventually develop resistance to imatinib mesylate and to other receptor tyrosine kinase inhibitors currently applied in the clinic. ${ }^{7}$ This resistance is mainly due to the re-activation of KIT signaling by the acquisition of secondary KIT mutations. The PI3K/AKT/mTOR signaling pathway represents a crucial driving force for the growth, survival, and progression of GIST. $^{8}$ In human malignancies, PTEN inactivation or insufficiency constitutively activates this pathway. ${ }^{9}$ Thereby, GIST patients with PTEN deficiency could benefit from alternative therapies targeting the PI3K/AKT/mTOR pathway. In line with this concept, an oral mTOR inhibitor everolimus (RAD001, Novartis Pharmaceuticals) has been tested in combination with imatinib in phase I-II clinical trials for patients with imatinib-resistant GIST, pointing to a potential therapeutic benefit of the combined administration. ${ }^{10}$ Recent preclinical in vivo studies performed on GIST xenografts, using PI3K inhibitors in combination with imatinib, indicated synergistic and long-lasting effects, and suggested that PTEN inactivation could have an impact on the response to this therapy. ${ }^{11,12}$ Yet, currently no studies have been performed to investigate the range of PTEN abnormities in imatinib-resistant GIST patients.

In this study, we have performed a comprehensive molecular and immuno-histochemical characterization of PTEN in a heterogeneous cohort of GIST, to assess the incidence and the nature of PTEN malfunction during GIST progression and during the course of imatinib therapy. Furthermore, we have investigated the impact of small interfering RNA (siRNA) PTEN knockdown on KIT signaling in imatinib-sensitive and imatinib-resistant GIST cell lines treated with a dual PI3K/mTOR inhibitor alone or in combination with imatinib, in order to better understand the functional consequences of PTEN insufficiency in GIST cells.

\section{Materials and methods}

Pathologic GIST specimens, from patients who underwent a biopsy or surgical resection of their tumor at the University Hospitals of Leuven, Belgium, and at the Department of Soft Tissue/ Bone Sarcoma and Melanoma M. Sklodowska-Curie Memorial Cancer Center and Institute of Oncology, Warsaw, Poland, were retrieved from the Departments of Pathology of both institutions. Patients with advanced GIST were treated with imatinib in a dose 400-800 mg per day. The majority of these patients acquired resistance to imatinib, which was clinically defined as progressive tumor growth that occurred after an initial period $>6$ months on imatinib during which the patient had responsive disease. Both response and progression have been objectively assessed according to RECIST criteria. Clinical information was obtained from the databases and from review of medical charts. The local institutional ethics board of both participating institutions approved the study.

In total, 112 specimens from imatinib-naïve ( $n=58,49$ primary and 7 metastatic) and imatinibtreated $(n=54 ; 48$ imatinib-progressive and 6 imatinib-responsive) GIST were available for the analysis (Table 1).

The pathologic material was examined and the diagnosis of GIST was confirmed using hematoxylin and eosin staining and CD117 (KIT) immunohistochemistry on formalin-fixed, paraffin-embedded tissues, according to standard procedures. KIT/ PDGFRA genotyping was done as reported. ${ }^{13}$

\section{PTEN Copy Number Loss, Mutational Analysis, and Methylation Status}

Dual-color interphase fluorescence in situ hybridization (FISH) was performed on 85 paraffinembedded specimens, using LSI PTEN(10q23)/ CEP10 Probe (Abbott Laboratories, Green Oaks, IL, USA), as described. ${ }^{12}$ The array comparative genomic hybridization (aCGH) data from 54 GIST included in this study were published before by our group. ${ }^{14,15}$

Mutational analysis of the entire coding sequence of PTEN (ENST00000371953-exons 1-9) was performed as described. ${ }^{12}$ The primer sequences are listed in Table 2.

The methylation status of the PTEN promoter (CpG Island 101755) was evaluated using the EpiTect Methyl qPCR Assay (Qiagen) according to the manufacturer's protocol. Universal Methylated Human DNA Standard (Zymo Research, Freiburg, Germany) and Human Genomic DNA (Roche, Basel, Switzerland) were used as positive and negative controls, respectively.

\section{PTEN Expression Analysis}

For the reverse-transcriptase quantitative PCR (RT-qPCR), total RNA ( $1 \mu \mathrm{g})$ was reverse transcribed with SuperScript III (Life Technologies, Carlsbad, CA, USA). The CDNA product was amplified using qPCR MasterMixPlus for SYBR ${ }^{\circledR}$ Green I without UNG (Eurogentec, Seraing, Belgium) in a Light Cycler 480 (Roche). The endogenous reference $G A D P H$ gene and the normal stomach tissues were used as references to normalize the results. PTEN 
Table 1 Clinical, histopathologic, and molecular findings of 112 GIST under study

\begin{tabular}{|c|c|c|c|c|c|c|c|c|c|c|c|c|c|c|}
\hline \multirow[b]{2}{*}{ No. } & \multirow[b]{2}{*}{$\begin{array}{c}\text { Age } \\
\text { (years) }\end{array}$} & \multirow[b]{2}{*}{ Gender } & \multicolumn{3}{|c|}{ Primary tumor } & \multirow[b]{2}{*}{$\begin{array}{l}\text { Type of tissue at the } \\
\text { time of surgery }\end{array}$} & \multirow[b]{2}{*}{ Primary genotype } & \multirow[b]{2}{*}{$\begin{array}{l}\text { Secondary } \\
\text { mutation }\end{array}$} & \multicolumn{2}{|c|}{ PTEN loss } & \multicolumn{2}{|c|}{$\begin{array}{c}\text { PTEN } \\
\text { expression }\end{array}$} & \multirow[b]{2}{*}{$\begin{array}{l}\text { PTEN } \\
\text { methylation }\end{array}$} & \multirow[b]{2}{*}{$\begin{array}{l}\text { PTEN } \\
\text { mutation }\end{array}$} \\
\hline & & & $\begin{array}{l}\text { Primary } \\
\text { site }\end{array}$ & $\begin{array}{l}\text { Size } \\
(\mathrm{mm})\end{array}$ & $\begin{array}{c}M I(\text { per } 50 \\
\text { HPF) }\end{array}$ & & & & $a C G H$ & FISH & $\begin{array}{l}R T- \\
P C R\end{array}$ & $I H C$ & & \\
\hline 1 & 65 & M & Colon & 25 & 35 & IM-PD & KIT 11 p.D579del & Not detected & ND & Yes & 0.1 & 0 & Negative & ND \\
\hline 2 & 59 & $\mathrm{~F}$ & Stomach & 100 & 52 & IM-PD & KIT 11 p.Q556_V559delinsH & KIT 13 p.V654A & ND & Yes & 0.38 & 0 & Negative & Negative \\
\hline 3 & 54 & $\mathrm{~F}$ & Intraabd & NA & NA & IM-PD & W' & KIT ampl & ND & Yes & ND & 0 & Negative & ND \\
\hline 4 & 42 & M & Stomach & 100 & NA & IM-PD & KIT 11 p.W557_K558del & KIT 14 p.T670I & ND & Yes & ND & 0 & Negative & Negative \\
\hline 5 & 47 & $\mathrm{~F}$ & Sm Int & 10 & 7 & IM-PD & KIT 11 p.V5̄59D & KIT: p.D820G & ND & Yes & 0.55 & 0 & Negative & Negative \\
\hline 6 & 49 & M & Stomach & 65 & 120 & IM-PD & KIT 11 p. K558_G565delinsR & Not detected & ND & Yes (homo) & 0.04 & 0 & ND & ND \\
\hline 7 & 51 & M & Sm Int & 12 & $>10$ & IM-PD & KIT 11 p.K550_K558del & KIT 17 p.D820Y & ND & Yes & 0.1 & 0 & Negative & ND \\
\hline 8 & 70 & M & Stomach & NA & NA & IM-PD & KIT 11 p.W557_K5̄58del homo & KIT 14 p.T670I & ND & No & ND & 0 & Negative & ND \\
\hline 9 & 39 & M & Duod & NA & NA & IM-PD & KIT 11 p.W557_K558del & Not detected & ND & Yes & ND & 0 & Negative & ND \\
\hline 10 & 55 & $\mathrm{M}$ & Colon & NA & NA & IM-PD & KIT 11 p.V569_L576del & Not detected & ND & Yes & 0.01 & 0 & Negative & Negative \\
\hline 11 & 47 & M & Sm Int & NA & 38 & IM-PD & KIT 9 p.A502_Y̌503dup & Not detected & ND & No & 0.95 & 1 & Negative & Negative \\
\hline 12 & 59 & M & Sm Int & NA & NA & IM-PD & KIT 11 p.L̄576P & KIT 17 p.D820Y & ND & Yes & 0.43 & 1 & Negative & Negative \\
\hline 13 & 40 & M & Intraab & 240 & 25 & IM-PD & KIT 11 p.V559G & KIT 13 p.V654A & Yes & Yes & ND & 1 & Negative & Negative \\
\hline 14 & 67 & M & Sm Int & 40 & 60 & IM-PD & KIT 11 p.K550_K558delinsQ & KIT 17 p.D820Y & ND & No & 1.75 & 1 & Negative & ND \\
\hline 15 & 45 & M & Sm Int & 60 & 28 & IM-PD & KIT 11 p.W557_T574del homo & KIT 17 p.N822K & ND & Yes & ND & 1 & Negative & Negative \\
\hline 16 & 57 & $\mathrm{~F}$ & Stomach & 150 & NA & IM-PD & KIT 11 p.P57̄3_T574dup & KIT 13 p.V654A & ND & Yes & ND & 1 & Negative & Negative \\
\hline 17 & 45 & $\mathrm{M}$ & Sm Int & 9 & 40 & IM-PD & KIT 11 p.N567_L̄576delinsI & Not detected & ND & No & 2.5 & 1 & Negative & ND \\
\hline 18 & 52 & $\mathrm{~F}$ & Sm Int & NA & 25 & IM-PD & KIT 9 p.A502_Y503dup & Not detected & ND & Yes & 0.54 & 1 & Negative & Negative \\
\hline 19 & 45 & M & Sm Int & 35 & 75 & IM-PD & KIT 11 p.W557_K558del & KIT 17 p.D816G & No & No & 1.34 & 1 & Negative & ND \\
\hline 20 & & & Colon meta & 13 & 20 & Met & KIT 11 p.W557_K558del & ND & No & No & ND & 1 & Negative & ND \\
\hline 21 & 59 & $\mathrm{~F}$ & Sm Int & 8 & 3 & IM-PD & KIT 9 p.A502_Y̌503dup & Not detected & ND & Yes & ND & 1 & Negative & ND \\
\hline 22 & 33 & $\mathrm{~F}$ & Stomach & Multiple & 46 & IM-PD & WT & Not detected & ND & Yes & 0.3 & 1 & Negative & ND \\
\hline 23 & 46 & M & Sm Int & $\mathrm{NA}$ & NA & IM-PD & KIT 11 p.K558delinsNP & Not detected & ND & Yes & ND & 1 & Negative & ND \\
\hline 24 & 64 & $\mathrm{M}$ & Sm Int & 40 & 15 & IM-PD & KIT 9 p.A502_Y503dup & Not detected & No & No & ND & 1 & ND & ND \\
\hline 25 & 65 & $\mathrm{M}$ & Rectum & NA & 47 & IM-PD & KIT 11 p.W557_V559delinsF & Not detected & ND & Yes & 0.31 & 1 & Negative & Negative \\
\hline 26 & 38 & $\mathrm{~F}$ & Sm Int & 90 & 9 & IM-PD & KIT 11 p.Q556_E561delinsQ & Not detected & No & No & 0.97 & 2 & Negative & ND \\
\hline 27 & 49 & M & Colon & 75 & 15 & IM-PD & KIT 11 p.M552_E554del & BRAF V600E & ND & No & ND & 2 & Negative & Negative \\
\hline 28 & 72 & M & Rectum & NA & 4 & IM-PD & KIT 11 p.K̄̄58N & BRAF V600E & ND & No & ND & 2 & ND & ND \\
\hline 29 & 58 & $\mathrm{M}$ & Colon & $>10$ & $>10$ & IM-PD & KIT 9 p.A502_Y503dup & Not detected & ND & No & 1.05 & 2 & ND & ND \\
\hline 30 & 63 & $\mathrm{~F}$ & Sm Int & 12 & 10 & IM-PD & KIT 9 p.A502_Y503dup & Not detected & ND & No & 0.93 & 2 & Negative & ND \\
\hline 31 & 56 & $\mathrm{~F}$ & Stomach & 95 & 10 & IM-PD & WT & Not detected & ND & No & 1.8 & 2 & ND & ND \\
\hline 32 & 56 & M & Sm Int & 10 & 14 & IM-PD & KIT 9 p.A502_Y503dup & Not detected & ND & No & 1.21 & 2 & Negative & Negative \\
\hline 33 & 43 & M & Stomach & 8 & 15 & IM-PD & KIT 11 p.W557_K558del hom & KIT 13 p.V654A & ND & No & 1.56 & 2 & Negative & ND \\
\hline 34 & 55 & $\mathrm{~F}$ & Sm Int & 70 & 11 & IM-PD & KIT 11 p.W557R & KIT 17 p.N822K & No & No & 1.19 & 2 & Negative & Negative \\
\hline 35 & 51 & $\mathrm{M}$ & Sm Int & 15 & 0 & IM-PD & KIT 11 p.W557_K558del & KIT 17 p.N822K & ND & No & 1.25 & 2 & ND & ND \\
\hline 36 & 76 & M & Sm Int & NA & 55 & IM-PD & KIT 9 p.A502_Y̌503dup & Not detected & ND & No & 0.53 & 3 & Negative & ND \\
\hline 37 & 50 & $\mathrm{~F}$ & Stomach & NA & 52 & IM-PD & WT & Not detected & No & No & 3.79 & 3 & ND & ND \\
\hline 38 & 41 & $\mathrm{M}$ & Colon & 38 & 14 & IM-PD & KIT 9 p.A502_Y503dup & Not detected & No & No & 1.23 & 3 & ND & ND \\
\hline 39 & & & Colon & 80 & 2 & Met & KIT 9 p.A502_Y503dup & ND & No & ND & 1.23 & 3 & Negative & Negative \\
\hline 40 & 44 & M & Intraab & NA & 25 & IM-PD & KIT 9 p.A502_Y503dup & KIT 13 p.V654A & ND & No & 1.36 & 3 & Negative & Negative \\
\hline 41 & 12 & $\mathrm{~F}$ & Stomach & Multiple & 25 & IM-PD & WT & Not detected & No & No & ND & 3 & ND & ND \\
\hline 42 & 22 & $\mathrm{~F}$ & Stomach & 230 & 51 & IM-PD & KIT 11 p.I563_Q575del & KIT 17 p.D820Y & ND & No & ND & 3 & ND & ND \\
\hline 43 & 41 & $\mathrm{~F}$ & Sm Int & 150 & 17 & IM-PD & KIT 9 p.A502_Y503dup & Not detected & ND & Yes & 0.49 & 3 & ND & ND \\
\hline 44 & 66 & $\mathrm{~F}$ & Stomach & 50 & 126 & IM-PD & PDGFRA 18 p.D842V & Not detected & ND & No & 3.16 & 3 & ND & ND \\
\hline 45 & 56 & M & Sm Int & 10 & 100 & IM-PD & KIT 11 p.E554_D572del & KIT 13 p.V654A & ND & No & 1.9 & 3 & ND & ND \\
\hline 46 & 54 & M & Sm Int & 16 & 20 & IM-PD & KIT 9 p.A502_Y503dup & Not detected & ND & No & 1.63 & 3 & Negative & Negative \\
\hline 47 & 56 & M & Sm Int & 2 & 10 & IM-PD & KIT 11 p.V560E homo & Not detected & ND & No & 0.86 & 3 & ND & ND \\
\hline 48 & 73 & M & Sm Int & 10 & 60 & IM-PD & KIT 11 p.V560D & KIT 13 p.V654A & ND & No & ND & 3 & ND & ND \\
\hline 49 & 37 & $\mathrm{~F}$ & Stomach & NA & NA & IM-PD & WT & Not detected & ND & Yes & 0.21 & 3 & ND & ND \\
\hline 50 & 43 & $\mathrm{~F}$ & Sm Int & 6 & 15 & IM-PD & KIT 9 p.A502_Y503dup & Not detected & ND & No & 1.6 & 3 & Negative & Negative \\
\hline 51 & 50 & M & Sm Int & 7 & 2 & IM-RESP & KIT 11 p.M552_W557del & Not detected & ND & No & 2.08 & 1 & Negative & Negative \\
\hline
\end{tabular}




\begin{tabular}{|c|c|c|c|c|c|c|c|c|c|c|c|c|c|c|c|}
\hline \multirow[b]{2}{*}{ No. } & \multirow[b]{2}{*}{$\begin{array}{c}\text { Age } \\
\text { (years) }\end{array}$} & \multirow[b]{2}{*}{ Gender } & \multicolumn{3}{|c|}{ Primary tumor } & \multirow[b]{2}{*}{$\begin{array}{l}\text { Type of tissue at the } \\
\text { time of surgery }\end{array}$} & \multirow[b]{2}{*}{ Primary genotype } & \multirow[b]{2}{*}{$\begin{array}{l}\text { Secondary } \\
\text { mutation }\end{array}$} & \multicolumn{2}{|c|}{ PTEN loss } & \multicolumn{2}{|c|}{$\begin{array}{c}\text { PTEN } \\
\text { expression }\end{array}$} & \multirow[b]{2}{*}{$\begin{array}{l}\text { PTEN } \\
\text { methylation }\end{array}$} & \multirow[b]{2}{*}{$\begin{array}{l}\text { PTEN } \\
\text { mutation }\end{array}$} & \\
\hline & & & $\begin{array}{l}\text { Primary } \\
\text { site }\end{array}$ & $\begin{array}{c}\text { Size } \\
(\mathrm{mm})\end{array}$ & $\begin{array}{c}M I(\text { per } 50 \\
H P F)\end{array}$ & & & & $a C G H$ & FISH & $\begin{array}{l}R T- \\
P C R\end{array}$ & IHC & & & \\
\hline 52 & 34 & $\mathrm{~F}$ & Stomach & 6 & 10 & IM-RESP & WT & Not detected & ND & No & ND & 2 & Negative & ND & \\
\hline 53 & 47 & $\mathrm{~F}$ & Sm Int & 12 & 1 & IM-RESP & KIT 11 p.K558_G565delinsR & Not detected & ND & ND & 2.43 & 1 & ND & ND & \\
\hline 54 & 33 & $\mathrm{M}$ & Sm Int & $>10$ & 12 & IM-RESP & KIT 11 p.V559_Y568delinsDND & Not detected & ND & Yes & ND & 1 & Negative & ND & \\
\hline 55 & 19 & $\mathrm{~F}$ & Stomach & 25 & 10 & IM-RESP & PDGFRA ex4 & Not detected & ND & No & ND & 2 & Negative & ND & \\
\hline 56 & 49 & $\mathrm{~F}$ & Sm Int & NA & NA & IM-RESP & KIT 11 p.W557_K558del & Not detected & ND & No & 1.75 & 2 & Negative & Negative & \\
\hline 57 & 74 & $\mathrm{M}$ & Stomach & 90 & 200 & Met & KIT 11 p.V560D & ND & Yes & Yes & 0.4 & 1 & Negative & Negative & \\
\hline 58 & 63 & $\mathrm{~F}$ & Sm Int & NA & 21 & Met & KIT 9 p.A502_Y503dup & ND & ND & No & 0.82 & 1 & Negative & Negative & \\
\hline 59 & 61 & $\mathrm{~F}$ & $\begin{array}{l}\text { Liver, } \\
\text { primary } \\
\text { unknown }\end{array}$ & NA & 55 & Met & $\begin{array}{c}\text { PDGFRA } 18 \text { p.R841_M844delinsR } \\
\text { homo }\end{array}$ & ND & Yes & ND & ND & 2 & ND & ND & \\
\hline 60 & 61 & $\mathrm{M}$ & Sm Int & 80 & 5 & Met & KIT 17 p.N822K & ND & No & No & 2.23 & 2 & Negative & Negative & \\
\hline 61 & 78 & $\mathrm{M}$ & Sm Int & 280 & 5 & Met & KIT 11 p.W557_V559delinsF & ND & No & No & 0.74 & 1 & Negative & ND & \\
\hline 62 & 25 & $\mathrm{~F}$ & Stomach & 170 & 31 & High & PDGFRA 18 p.D842_H845del homo & o ND & No & No & ND & 1 & ND & ND & \\
\hline 63 & 48 & $\mathrm{~F}$ & Stomach & 70 & 14 & High & PDGFRA 18 p.D842V & ND & No & ND & ND & 1 & ND & ND & \\
\hline 64 & 61 & $\mathrm{M}$ & Sm Int & 38 & 7 & High & KIT 9 p.A502_Y503dup & ND & No & No & 1.02 & 1 & ND & ND & \\
\hline 65 & 68 & $\mathrm{M}$ & Sm Int & 76 & 8 & High & KIT 11 p.M552_E554delinsK & ND & No & No & ND & 1 & Negative & Negative & \\
\hline 66 & 57 & $\mathrm{M}$ & Stomach & $>10$ & 23 & High & KIT 11 p.W557_V559delinsF & ND & No & No & 1.45 & 1 & ND & ND & \\
\hline 67 & 65 & $\mathrm{M}$ & Sm Int & 150 & 17 & High & KIT 9 p.A502_Y503dup & ND & No & ND & ND & 2 & Negative & Negative & \\
\hline 68 & 65 & $\mathrm{~F}$ & Stomach & 180 & 60 & High & KIT 11 p.W557_V560delinsF & ND & No & No & 2.0 & 3 & ND & ND & \\
\hline 69 & 69 & $\mathrm{M}$ & Mesent & 120 & 21 & High & KIT 11 p.V560D & ND & ND & Yes & ND & 3 & Negative & Negative & \\
\hline 70 & 48 & $\mathrm{~F}$ & Stomach & 50 & 37 & High & KIT 11 p.K550-V555del & ND & ND & No & 1.33 & 3 & ND & ND & \\
\hline 71 & 59 & $\mathrm{M}$ & Sm Int & 35 & 7 & High & KIT 11 p.V560_L576delinsD & ND & No & ND & ND & 3 & Negative & Negative & \\
\hline 72 & 78 & $\mathrm{M}$ & Sm Int & 55 & 12 & High & KIT 11 p.V560D & ND & ND & No & 1.03 & 3 & ND & ND & $>1$ \\
\hline 73 & 72 & $\mathrm{M}$ & Oesoph & 20 & 11 & High & KIT 11 p.K558_V559delinsN homo & ND & No & No & 1.0 & 3 & ND & ND & ? \\
\hline 74 & 44 & $\mathrm{~F}$ & Stomach & 160 & 4 & High & KIT 11 p.W557R & ND & Yes & Yes & 0.84 & 3 & ND & ND & $\stackrel{0}{\rightleftarrows}$ \\
\hline 75 & 69 & $\mathrm{M}$ & Stomach & 40 & 11 & Intermed & PDGFRA 18 p.D842_H845del & ND & No & ND & ND & 2 & ND & ND & $\bar{\Xi}$ \\
\hline 76 & 83 & $\mathrm{~F}$ & Stomach & 85 & 7 & Intermed & KIT 11 p.V560D & ND & ND & No & 1.06 & 2 & ND & ND & $\begin{array}{c}0 \\
0 \\
0\end{array}$ \\
\hline 77 & 75 & $\mathrm{~F}$ & Stomach & 110 & 1 & Intermed & PDGFRA 18 p.D842V & ND & No & ND & ND & 2 & ND & ND & $\overline{2}$ \\
\hline 78 & 69 & $\mathrm{M}$ & Stomach & $>100$ & 4 & Intermed & KIT 11 p.L576_R588dup & ND & No & ND & 0.96 & 2 & ND & ND & \\
\hline 79 & 51 & $\mathrm{M}$ & Stomach & 130 & 2 & Intermed & PDGFRA $18 \mathrm{p} . D 842 \mathrm{~V}$ & ND & No & ND & ND & 2 & ND & ND & \\
\hline 80 & 76 & $\mathrm{~F}$ & Stomach & 30 & 17 & Intermed & PDGFRA 18 p.D842V & ND & ND & ND & 0.76 & 2 & Negative & Negative & \\
\hline 81 & 53 & $\mathrm{M}$ & Duod & 55 & 5 & Intermed & KIT 11 p.L576P & ND & No & No & 1.59 & 3 & ND & ND & \\
\hline 82 & 68 & $\mathrm{~F}$ & Duod & 30 & 4 & Intermed & KIT 11 p.V560A & ND & No & ND & 1.39 & 3 & ND & ND & \\
\hline 83 & 67 & $\mathrm{~F}$ & Stomach & 40 & 25 & Intermed & KIT 11 p.W557R & ND & No & No & 1.58 & 3 & ND & ND & \\
\hline 84 & 50 & $\mathrm{~F}$ & Sm Int & 50 & 5 & Intermed & KIT 9 p.A502_Y503dup & ND & No & No & 4.08 & 3 & ND & ND & \\
\hline 85 & 36 & $\mathrm{~F}$ & Stomach & 35 & 60 & Intermed & KIT 11 p.W557-K558del homo & ND & No & No & ND & 3 & ND & ND & \\
\hline 86 & 47 & $\mathrm{~F}$ & Duod & 60 & 2 & Intermed & KIT 11 p.P577_R588dup; p.L589S & ND & No & ND & ND & 3 & Negative & Negative & \\
\hline 87 & 77 & $\mathrm{~F}$ & Eosoph & 100 & 5 & Intermed & KIT 9 p.A502_Y503dup & ND & ND & ND & ND & 3 & $\mathrm{ND}$ & ND & \\
\hline 88 & 85 & $\mathrm{M}$ & Stomach & 60 & 5 & Low & KIT 11 p.V559A & ND & ND & No & 1.43 & 2 & ND & ND & \\
\hline 89 & 61 & $\mathrm{M}$ & Stomach & 55 & 5 & Low & KIT 11 p.V554D & ND & No & No & 2.29 & 2 & ND & ND & \\
\hline 90 & 53 & M & Stomach & 60 & 5 & Low & KIT 11 p.V559D & ND & ND & $\begin{array}{l}\text { gain of chr. } \\
10\end{array}$ & 1.74 & 2 & ND & ND & \\
\hline 91 & 49 & $\mathrm{M}$ & Stomach & 75 & 4 & Low & PDGFRA 18 p.D842V & ND & No & No & ND & 2 & Negative & Negative & \\
\hline 92 & 64 & $\mathrm{M}$ & Stomach & 75 & 6 & Low & KIT 11 p.W557_K558del & ND & ND & ND & 0.74 & 2 & ND & & \\
\hline 93 & 62 & $\mathrm{M}$ & Stomach & 90 & 5 & Low & PDGFRA 18 p.D842V & ND & No & ND & ND & 2 & Negative & Negative & \\
\hline 94 & 72 & M & Sm Int & 48 & 3 & Low & KIT 11 p.V560D & ND & No & No & 0.83 & 2 & ND & & \\
\hline 95 & 46 & $\mathrm{~F}$ & Stomach & 50 & 5 & Low & KIT 11 p.K558_G565delinsN & ND & Yes & No & ND & 2 & Negative & Negative & \\
\hline 96 & 80 & $\mathrm{~F}$ & Stomach & 55 & 3 & Low & KIT 11 p.581-590insKWEFPRNRLS & $S \mathrm{ND}$ & ND & No & ND & 2 & Negative & Negative & \\
\hline 97 & 55 & $\mathrm{M}$ & Stomach & 50 & 5 & Low & PDGFRA 14 p.N659K & ND & No & ND & ND & 3 & $\mathrm{ND}$ & ND & \\
\hline 98 & 68 & $\mathrm{M}$ & Stomach & 30 & 6 & Low & KIT 11 p.Q556_I563del & ND & No & No & 1.55 & 3 & ND & ND & \\
\hline 99 & 44 & $\mathrm{M}$ & Stomach & 80 & 5 & Low & PDGFRA 18 p.D842V & ND & No & ND & ND & 3 & ND & ND & \\
\hline
\end{tabular}






expression was considered abnormal if the $\Delta \Delta \mathrm{Ct}$ $P T E N / G A D P H$ value was $<0.6$.

The PTEN protein expression was assessed by immunohistochemistry in 112 GIST using antiPTEN antibody (DAKO, Glostrup, Denmark; dilution 1:1000) previously validated on GIST xenografts with a known PTEN status. ${ }^{12}$ Scoring for PTEN staining was semi-quantitative, based on the proportion and intensity of positive neoplastic cells (Figure 1). It was performed in parallel by two observers using a four-tier system, ranging from $3+$ ( $>50 \%$ immune-reactive cells and strong intensity equal to that of the vascular endothelium; Figures 1a and b), $2+(>50 \%$ immune-reactive cells and weak intensity of staining; Figures 1c and d), $1+(<50 \%$ immune-reactive cells, referred to as reduced staining; Figure 1e) to 0 (completely negative; Figure 1f). The categories 0 and $1+$ were considered to be abnormal.

\section{In vitro siRNA PTEN Experiments}

In vitro studies were performed on imatinibsensitive GIST-T1 (carrier of KIT-V560_Y579del mutation) and imatinib-resistant GIST430 (carrier of primary KIT-V560_L576del and secondary KIT-V654A mutations) cell lines.

The compounds, imatinib mesylate and the dual PI3K/mTOR inhibitor NVP-BEZ235, ${ }^{16}$ were purchased from Sequoia Research Products (Pangbourne, UK).

The siRNA experiments were performed by the use of predesigned stealth RNAi duplexes against human PTEN (PTEN Validated Stealth RNAi: $\quad$ PTENHSS183790 + PTENHSS183791 + PTENHSS183792; Invitrogen). Transient transfection was done in six-well plates at a density of $10^{6}$ cells/plate with use of metafectene transfection reagent (Biontex, Planegg, Germany), according to the manufacturer's instructions. After $96 \mathrm{~h}$, medium was exchanged for the one supplemented with dimethyl sulfoxide (Sigma-Aldrich, St Louis, MO, USA) only, or $100 \mathrm{nM}$ NVP-BEZ235, or $200 \mathrm{nM}$ imatinib, or the combination of both drugs in a given concentration. After $2 \mathrm{~h}$ of treatment, the cell pellets were collected for protein analysis by western blotting. The antibodies against total KIT and total PTEN (DAKO), total MAPK (Invitrogen), phospho-KIT, phospho-AKT, total AKT, phosphoMAPK, phospho-S6, total S6 (Cell Signaling, Beverly, MA), and B-actin (Sigma-Aldrich) were applied.

\section{Statistics}

For statistical analysis of the qPCR data, the Mann-Whitney $U$-test was applied. For analyses of the frequency of the genomic PTEN losses and the abnormal PTEN expression by immunohistochemistry, the $\chi^{2}$ or Fisher exact tests were applied. 
Table 2 Primers for PTEN mutational analysis and quantitative RT-PCR

\begin{tabular}{ll} 
Target & Sequence $\left(5^{\prime} \rightarrow 3^{\prime}\right)$ \\
\hline Amplicons for PTEN & mutation analysis \\
PTEN exon 1 & F: TTCCATCCTGCAGAAGAAGC \\
& R: CTACGGACATTTTCGCATCC \\
PTEN exon 2 & F: AGTATTCTTTAGTTTGATTGCTGCAT \\
& R: CACAAAGTATCTTTTCTGTGGCTTA \\
PTEN exon 3 & F: GAAAATCTGTCTTTTGGTTTTCTTG \\
& R: TGGACTTCTTGACTTAATCGGTTT \\
PTEN exon 4 & F: TCACATTATAAAGATTCAGGCAATGT \\
& R: GTATCTCACTCGATAATCTGGATGACT \\
PTEN exon 5 & F: CCTGTTAAGTTGTATGCAACATTTCT \\
& R: TCCAGGAAGAGGAAAGGAAAA \\
PTEN exon 6 & F: AATGGCTACGACCCAGTTACC \\
& R: TCAAATGCTTCAGAAATATAGTCTCCT \\
PTEN exon 7 & F: AATCCATATTTCGTGTATATTGCTGA \\
& R: CACCTGCAGATCTAATAGAAAACAAA \\
PTEN exon 8 & F: TGTCATTTCATTTCTTTTCTTTCTT \\
& R: AAGTCAACAACCCCACAAA \\
PTEN exon 9 & F: TGTTCATCTGCAAAATGGAATAAA \\
& R: CACAATGTCCTATTGCCATTAAAA \\
Amplicons for quantitative RT-PCR \\
PTEN ex 6/7 & F: CAATGTTCAGTGGCGGAACTT \\
PTEN ex 6-7/7 & F: TGAATTGGAGGAATATATCTTCACCTT \\
& R: TGGGTCCTGAATTGGAGGAA \\
FADPH & F: TGACACTGGCAAAACAATGCA \\
& R: GGTCCTTTTCACCAGCAAGCT \\
&
\end{tabular}

Differences with $P$-value $<0.05$ were defined as statistically significant. The software STATISTICA (Stat Soft, USA - version 9.0) was used for statistical calculations.

\section{Results}

PTEN Mono-Allelic Loss Occurs in the Progressive Stage of Disease and is Frequent in Imatinib-Treated Tumors

To assess the incidence of PTEN loss at the genomic level, we performed FISH analysis of 85 samples, and complemented these data with aCGH results available from 54 cases (Table 1). In total, loss of PTEN occurred in 26 out of 108 samples (24\%); all of those tumors presented mono-allelic loss of the PTEN locus except for one, characterized by PTEN nullisomy. Concurrent analysis of PTEN status by FISH and aCGH indicated concordance of the results in 30 out of 31 cases; only in one specimen, FISH failed to identify a PTEN deletion otherwise seen by aCGH.

Within the imatinib-naïve group, PTEN loss was observed less frequently in very low/low/intermediate risk $v S$ high risk/metastatic GIST $(P=0.03$; Table 3). Of note, the incidence of PTEN loss in imatinib-treated tumors was high (39\%). As we did not have baseline pre-treatment tumor specimens available for comparison, we could not discriminate whether PTEN loss added to acquired resistance to imatinib or it was only a reflection of a more advanced stage of disease. Nevertheless, there was no correlation between the incidence of PTEN loss and the presence of secondary imatinib-resistant KIT mutations in GIST refractory to imatinib $(P=0.6)$. Moreover, no significant differences were found between primary high risk/metastatic and imatinib-treated GIST $(P=0.11)$. These results argue against the hypothesis that PTEN loss would be a direct cause of resistance to imatinib.

To further elucidate possible mechanisms responsible for aberrant PTEN expression, we performed mutational analysis of PTEN in 36 specimens (including 19 imatinib-progressive GIST). No somatic mutations were detected. In addition, we assessed PTEN promoter methylation in a total of 57 samples (including 24 imatinib-resistant GIST that lacked or expressed low level of PTEN protein by immunohistochemistry). None of the examined tumors exhibited methylation of PTEN promoter.

\section{PTEN Mono-Allelic Loss Correlates with Altered PTEN Expression on Transcript and Protein Levels}

By RT-qPCR, we investigated the expression of PTEN transcripts in 67 GIST (including 36 imatinib-resistant tumors). Abnormal PTEN expression was detected in $21 \%$ of samples. The low level of PTEN transcript expression correlated well with PTEN loss on the genomic level $(P=0.002)$.

By immunohistochemistry, absent or reduced expression of PTEN protein was observed in $32 \%$ $(n=36)$ of the tumors (Table 3$)$. In the imatinibnaïve cohort, abnormal PTEN protein level was detected in high-risk/metastatic but not in low/ intermediate-risk tumors ( $45 \%$ vs $0 \% ; P<0.001)$. In imatinib-treated tumors, abnormal PTEN expression was common (50\%); notably, all 10 completely PTEN-immuno-negative GIST were from imatinibprogressive cohort. The abnormal PTEN protein expression correlated with PTEN loss at the genomic level $(P=0.001)$.

\section{siRNA-Induced Downregulation of PTEN Expression in GIST Cell Lines Results in PI3K-AKT-mTOR and MAPK Pathway Activations}

In the PTENsi GIST-T1 and GIST430 cells, the level of PTEN protein was decreased by $\sim 50 \%$ and $80 \%$, respectively, compared with non-PTENsi cells (Figure 2).

In IM-S GIST-T1, PTEN silencing resulted in overactivation of AKT and MAPK (by 1.6- and 1.8fold, respectively). The AKT hyper-phosphorylation was partially reverted by $100 \mathrm{nM}$ NVP-BEZ235 in both PTENsi and non-PTENsi cells (by 70\% and $85 \%$, respectively). As expected, exposure to $200 \mathrm{nM}$ imatinib led to complete AKT inactivation in non-PTENsi cells, opposite to PTENsi cells, in 

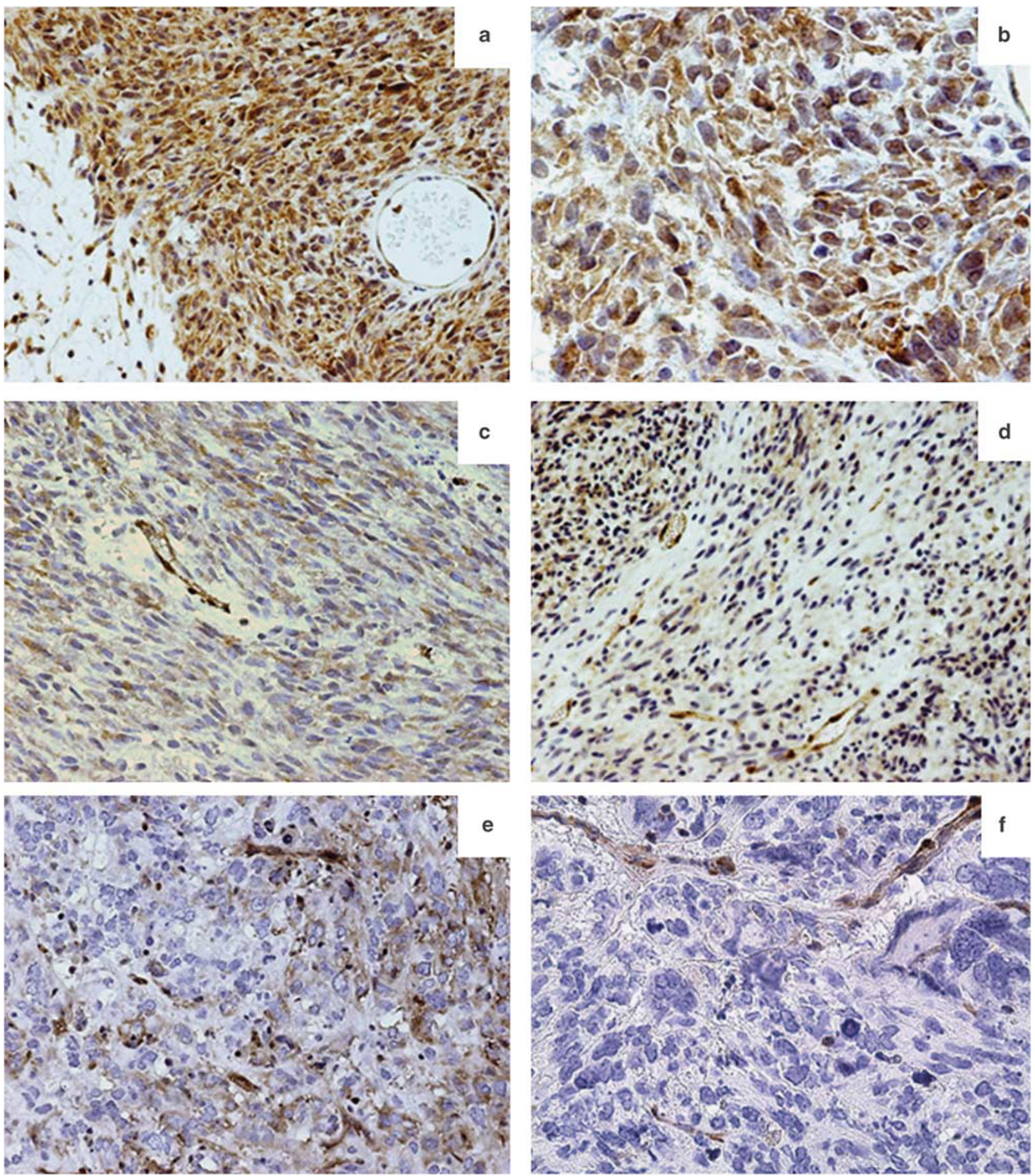

Figure 1 Examples of the PTEN immunostaining in GIST. Normal staining was defined as intense, cytoplasmic, and nuclear PTEN immunoreactivity in majority of tumor cells, with the staining intensity equal to that of the vascular endothelium (which served as internal positive control); original magnifications $\times 100$ (a) and $\times 400(\mathbf{b})$. The weaker reactivity of neoplastic cells in comparison with the vascular endothelium in $>50 \%$ of the neoplastic cells; original magnification $\times 200$ (c) and $\times 100$ (d). Reduced (e) or absent (f) PTEN expression in tumor specimens; original magnification $\times 200$. Immunostains counterstained with hematoxylin.

which still redundant AKT signaling existed, as proven by incomplete downstream p-S6 inhibition (sixfold higher in PTENsi vs non-PTENsi cells). This effect disappeared under the combined treatment regimen.
Interestingly, NVP-BEZ235 treatment gave rise to MAPK phosphorylation in non-PTENsi cells and at higher extent in PTENsi cells (2- and 2.8-fold, respectively). The MAPK activation was substantially and equally abolished by imatinib alone or 
Table 3 Correlation of PTEN loss by FISH/aCGH and PTEN protein expression by immunohistochemistry with clinicopathological data in GIST under study

\begin{tabular}{|c|c|c|c|c|c|c|c|c|c|c|c|}
\hline \multirow{3}{*}{ Categories } & \multicolumn{5}{|c|}{ PTEN by FISH/aCGH } & \multicolumn{6}{|c|}{ PTEN protein by immunohistochemistry } \\
\hline & \multirow{2}{*}{$\mathrm{n}$} & \multirow{2}{*}{ With loss } & \multirow{2}{*}{$\%$ Of total } & \multicolumn{2}{|l|}{ Statistical analysis } & \multirow{2}{*}{$\mathrm{n}$} & \multirow{2}{*}{ Absent } & \multirow{2}{*}{ Reduced } & \multirow{2}{*}{$\begin{array}{l}\text { Abnormal } \\
\% \text { of total }\end{array}$} & \multicolumn{2}{|l|}{ Statistical analysis } \\
\hline & & & & & P-value & & & & & & P-value \\
\hline Total & 108 & 26 & 24 & & & 112 & 10 & 26 & 32 & & \\
\hline \multicolumn{12}{|l|}{ Gender } \\
\hline Male & 63 & 14 & 22 & Male vs female & 0.59 & 64 & 7 & 18 & 39 & Male vs female & 0.07 \\
\hline Female & 45 & 12 & 27 & & & 48 & 3 & 8 & 22 & & \\
\hline \multicolumn{12}{|l|}{ Primary tumor site } \\
\hline Gastric & 52 & 9 & 17 & Gastric vs non-gastric & 0.11 & 54 & 4 & 6 & 18 & Gastric vs non-gastric & 0.002 \\
\hline Non-gastric & 56 & 17 & 30 & & & 58 & 6 & 20 & 45 & & \\
\hline \multicolumn{12}{|l|}{ GIST category } \\
\hline Imatinib naïve $\mathrm{a}^{\mathrm{a}}$ & 55 & 5 & 9 & Imatinib naïve $v s$ imatinib treated & $<0.001$ & 58 & 0 & 9 & 16 & Imatinib naïve $v s$ imatinib treated & $<0.001$ \\
\hline V. low/Low risk & 24 & 1 & 4 & High risk/meta vs imatinib treated & 0.11 & 25 & 0 & 0 & 0.0 & High/meta vs imatinib treated & 0.7 \\
\hline Inter. risk & 11 & 0 & 0 & High risk/meta vs low/Inter. risk & 0.03 & 13 & 0 & 0 & 0.0 & High/meta vs V. low/low/Inter. risk & $<0.001$ \\
\hline High risk & 13 & 2 & 15 & High risk vs meta & 0.48 & 13 & 0 & 5 & 38 & High/Inter. vs low risk & 0.02 \\
\hline Meta & 7 & 2 & 28 & & & 7 & 0 & 4 & 57 & High vs Inter./low risk & $<0.001$ \\
\hline Imatinib treated & 53 & 21 & 40 & & & 54 & 10 & 17 & 50 & & \\
\hline Mutation status & & & & & & & & & & WT vs PDGFRA & 0.2 \\
\hline KIT mutants & 83 & 22 & 26 & KIT 9 vs KIT 11 mutants & 0.2 & 58 & 9 & 23 & 37.2 & KIT 9 vs KIT 11 & 0.5 \\
\hline PDGFRA mutants & 18 & 1 & 6 & KIT vs PDGFRA mutants & 0.054 & 19 & 0 & 2 & 10.5 & KIT vs PDGFRA & 0.02 \\
\hline KIT/PDGFRA-WT & 7 & 3 & 43 & KIT mutants vs WT & 0.3 & 7 & 1 & 1 & 28.5 & KIT mutants vs WT & 0.6 \\
\hline With secondary KIT mutation & 21 & 9 & 43 & $\begin{array}{l}\text { With secondary KIT mutation } \\
\text { vs without }\end{array}$ & 0.6 & 21 & 6 & 6 & 57.1 & $\begin{array}{l}\text { With secondary KIT mutation } \\
\text { vS without }\end{array}$ & 0.4 \\
\hline
\end{tabular}

Abbreviations: aCGH, array comparative genomic hybridization; FISH, fluorescence in situ hybridization; Inter., intermediate; Meta, metastasis; V. low, very low; WT, wild type.

${ }^{\text {a}}$ Risk of recurrence for primary imatinib naive GIST was assessed according to the Armed Forces Institute of Pathology criteria. ${ }^{23}$ 
a

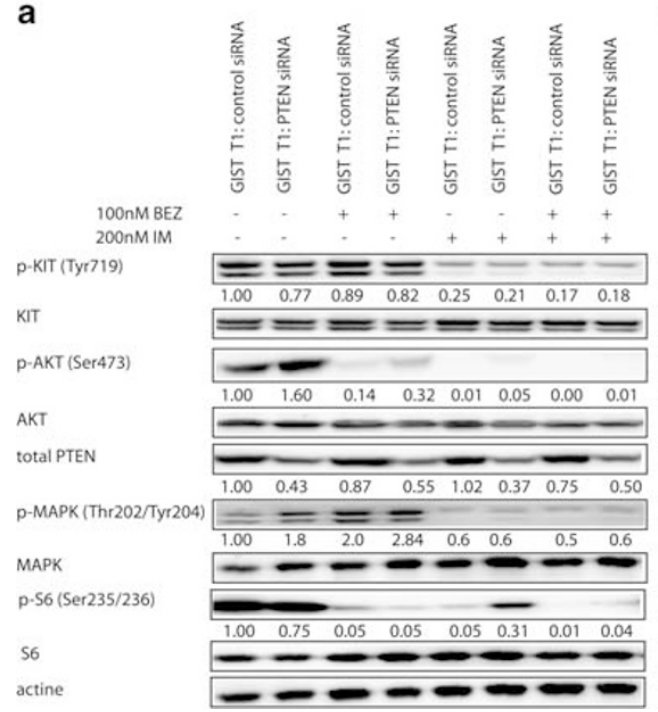

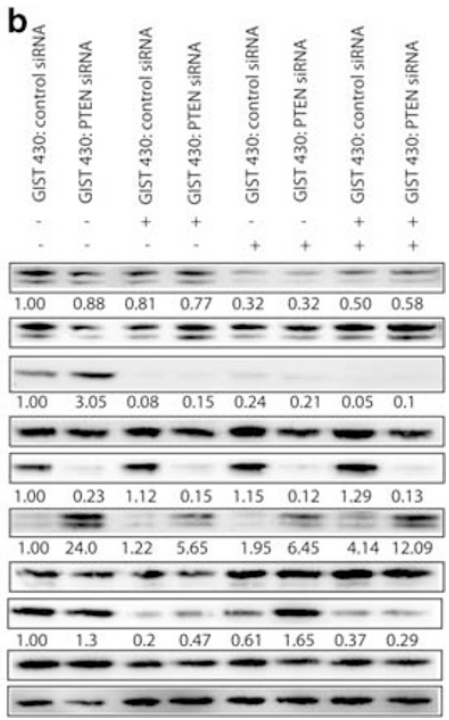

Figure 2 Short interfering (siRNA) knockdown of PTEN in imatinib-sensitive GIST-T1 (a) and imatinib-resistant GIST430 (b) cell lines. The effect of PTEN silencing on KIT downstream signaling was evaluated by western blotting under DMSO, imatinib, or NVP-BEZ235 alone, or combined treatment. For densitometry analysis, bands were normalized to actin expression and compared with control (diluting medium) as previously described. ${ }^{12}$

under the combined treatment in both, non-PTENsi and PTENsi cells.

In imatinib-resistant GIST430 cells, knockdown of PTEN induced the increase of p-AKT (3-fold) and even more remarkable of p-MAPK (24-fold). The AKT phosphorylation was significantly inhibited under NVP-BEZ235 or imatinib treatment alone or combination in PTENsi and in non-PTENsi cells. In contrast, MAPK hyperactivation in PTENsi cells was still substantially higher compared with control cells under both NVP-BEZ235 (5.6-fold) and imatinib (6.4-fold). Noteworthy, combined treatment of NVP-BEZ235 with imatinib led still to a 4- and 12fold overactivation of MAPK in non-PTENsi and PTENsi cells, respectively. The S6 protein, downstream intermediate of the mTOR pathway, was overactivated by $30 \%$ in PTENsi cells in comparison with control. The NVP-BEZ235 treatment resulted in reduction of S6 phosphorylation in non-PTENsi and, to a lesser extent, in PTENsi cells (80 and 50\%, respectively). Imatinib treatment induced $40 \%$ reduction of p-S6 in control cells; in contrast, a $60 \%$ increase in the level of p-S6 was observed in PTENsi cells. Markedly, this overactivation of S6 protein in PTENsi vs non-PTENsi cells was only partially reverted by combined treatment.

\section{Discussion}

First, we carried out FISH and/or aCGH analysis of PTEN in a heterogeneous cohort of GIST. Monoallelic PTEN loss occurred in $24 \%$ of cases, whereas bi-allelic PTEN loss was encountered only in one tumor. In the imatinib-naïve GIST, a positive correlation between mono-allelic loss of PTEN and high-risk/metastatic tumors were found $(P=0.03)$. These findings are in agreement with aCGH analysis reported by Ylipää et al, ${ }^{17}$ as they observed the loss of chromosome 10q (on which PTEN maps) in the specific GIST subgroup, in patients with poor clinical outcome. As such, this event is likely to occur in the late stage of GIST evolution. As revealed by RT-qPCR analysis, PTEN loss was associated with substantially lower or absent PTEN transcript levels $(P=0.002)$. In contrast to Yang et al., ${ }^{18}$ we do not have evidence that receptor tyrosine kinase inhibitor treatment can cause loss of PTEN expression by epigenetic silencing, as we did not identify PTEN promoter methylation in any of the analyzed imatinib-resistant GIST. This finding together with the lack of evidence for inactivation of the PTEN by inactivating mutations suggests that the aberrantly low PTEN expression in GIST might be mainly due to mono-allelic loss.

Our findings were corroborated at the protein level by immunohistochemical analysis. In the imatinib-naïve cohort, reduced PTEN expression was mainly associated with the high-risk/metastatic tumors $(P<0.001)$. An inverse correlation between PTEN immunoreactivity and disease progression in primary GIST has been previously reported. ${ }^{19,20}$ In our imatinib-treated GIST, the frequency of tumors with reduced or absent PTEN expression reached $50 \%$. This result is notable because to the best of our knowledge data about the incidence of PTEN deficiency in imatinib-treated GIST has not been reported as of yet. Importantly, a subset of imatinib-progressive GIST with mono-allelic PTEN loss showed complete lack of PTEN reactivity by 
immunohistochemistry, suggesting a bi-allelic inactivation. In these cases, we cannot exclude the presence of microdeletions/rearrangements of the second PTEN allele that were under the detection limit of the techniques used in the current study. Alternatively, other inactivation mechanisms might have a role, as PTEN expression could be positively and negatively regulated by transcription factors or microRNAs, as well as posttranslationally regulated by phosphorylation, oxidation, and acetylation. ${ }^{1,2}$ Downregulation of PTEN results in hyperactivation of the PI3K/AKT/ mTOR pathway, leading to proliferative advantage of neoplastic cells. A similar role was attributed to PTEN in other types of cancers. ${ }^{9}$

Subsequently, we explored the effect of siRNA PTEN silencing on KIT downstream signaling in vitro using GIST cell lines. Noteworthy, in both imatinib-sensitive and imatinib-resistant GIST cells, PTEN silencing resulted in overactivation of both, AKT and MAPK; the latter being exceptionally hyper-phosphorylated in imatinib-resistant cells. Overactivation of AKT was expected, as PTEN is a negative regulator of the PI3K-AKT pathway, whereas the latter might be explained by the extensive mTOR-negative feedback loops and cross-talks between the PI3K and the RAS-mediated MAPK pathways that have been well documented among the signaling networks driving tumor progression. ${ }^{21,22}$

Finally, we have investigated the effect of PI3K/ mTOR inhibition, alone or in combination with imatinib, on PTENsi GIST cell lines.

In imatinib-sensitive GIST-T1, NVP-BEZ235 treatment resulted in only partial inactivation of AKT in PTENsi cells, whereas enhancing the activation of MAPK. Nevertheless, the effect of PTEN silencing on AKT and MAPK activation in these cells was counteracted by imatinib alone and even more substantially by the combined treatment of NVPBEZ235 and imatinib.

In imatinib-resistant GIST-430 cells, NVP-BEZ235 treatment led to less efficient AKT and MAPK inhibition in PTENsi in comparison to non-PTENsi cells. Likewise, imatinib did not inhibit sufficiently the overactivated MAPK and downstream S6 proteins in PTENsi cells. Combined treatment resulted in still substantial MAPK hyper-phosphorylation, and only partially counteracted S6 activation.

In summary, our data strongly support an important role for PTEN downregulation in GIST progression. Partial or total PTEN depletion occurs frequently in imatinib-resistant GIST. In vitro studies suggest that PTEN insufficiency leads to upregulation of the PI3K/AKT and MAPK pathways. Depending on the molecular context of the individual tumors, the MAPK activity might be even paradoxically further enhanced under certain dualspecific $\mathrm{PI} 3 \mathrm{~K} / \mathrm{mTOR}$ inhibitors. Our results highlight the importance of molecularly sub-classifying GIST before exposing patients to innovative targeted treatments.

\section{Acknowledgments}

The GIST-T1 and GIST-430 cell lines were a kind gift from Dr Takahiro Taguchi (Kochi University, Japan) and Dr Jonathan A. Fletcher (Harvard Medical School, Boston, MA, USA), respectively. This work was supported by a Concerted Action Grant from the KU Leuven (GOA/11/2010), Research Grant from Life Raft Group, and KBN N N402 209235 Grant.

\section{Disclosure/conflict of interest}

P Schöffski received research funding and honoraria for advisory functions from Novartis. P Rutkowski received honoraria from Novartis, Pfizer, and he served as a member of Advisory Board for Novartis and Bayer. All remaining authors have declared no conflict of interest.

\section{References}

1 Song MS, Salmena L, Pandolfi PP. The functions and regulation of the PTEN tumour suppressor. Nat Rev Mol Cell Biol 2012;13:283-296.

2 Zhang P, Chen J, Guo X. New insights into PTEN regulation mechanisms and its potential function in targeted therapies. Biomed Pharmacother 2012;66: 485-490.

3 Nagata Y, Lan KH, Zhou X, et al. PTEN activation contributes to tumor inhibition by trastuzumab, and loss of PTEN predicts trastuzumab resistance in patients. Cancer Cell 2004;6:117-127.

4 Wang MY, Lu KV, Zhu S, et al. Mammalian target of rapamycin inhibition promotes response to epidermal growth factor receptor kinase inhibitors in PTENdeficient and PTEN-intact glioblastoma cells. Cancer Res 2006;66:7864-7869.

5 Jhawer M, Goel S, Wilson AJ, et al. PIK3CA mutation/ PTEN expression status predicts response of colon cancer cells to the epidermal growth factor receptor inhibitor cetuximab. Cancer Res 2008;68:1953-1961.

6 Kokubo Y, Gemma A, Noro R, et al. Reduction of PTEN protein and loss of epidermal growth factor receptor gene mutation in lung cancer with natural resistance to gefitinib (IRESSA). Br J Cancer 2005;92:1711-1719.

7 Corless CL, Barnett CM, Heinrich MC. Gastrointestinal stromal tumours: origin and molecular oncology. Nat Rev Cancer 2011;11:865-878.

8 Bauer S, Duensing A, Demetri GD, et al. KIT oncogenic signaling mechanisms in imatinib-resistant gastrointestinal stromal tumor: PI3-kinase/AKT is a crucial survival pathway. Oncogene 2007;26:7560-7568.

9 Alimonti A, Carracedo A, Clohessy JG, et al. Subtle variations in PTEN dose determine cancer susceptibility. Nat Genet 2010;42:454-459.

10 Schöffski P, Reichardt P, Blay JY, et al. A phase I-II study of everolimus (RAD001) in combination with imatinib in patients with imatinib-resistant gastrointestinal stromal tumors. Ann Oncol 2010;10: 1990-1998.

11 Van Looy T, Wozniak A, Sciot R, et al. Efficacy of a phosphoinositol 3 kinase (PI3K) inhibitor in gastro- 
intestinal stromal tumor (GIST) models. J Clin Oncol (Suppl) 2012;30:10030.

12 Floris G, Wozniak A, Sciot R, et al. A potent combination of the novel PI3K Inhibitor, GDC-0941, with imatinib in gastrointestinal stromal tumor xenografts: long-lasting responses after treatment withdrawal. Clin Cancer Res 2013;19:620-630.

13 Debiec-Rychter M, Cools J, Dumez H, et al. Mechanisms of resistance to imatinib mesylate in gastrointestinal stromal tumors and activity of the PKC412 inhibitor against imatinib-resistant mutant. Gastroenterology 2005;128:270-279.

14 Wozniak A, Sciot R, Guillou L, et al. Array CGH analysis in primary gastrointestinal stromal tumors: cytogenetic profile correlates with anatomic site and tumor aggressiveness, irrespective of mutational status. Genes Chromosomes Cancer 2007;46: 261-276.

15 Lagarde P, Pérot G, Kauffmann A, et al. Mitotic checkpoints and chromosome instability are strong predictors of clinical outcome in gastrointestinal stromal tumors. Clin Cancer Res 2012;18:826-838.

16 Maira SM, Stauffer F, Brueggen J, et al. Identification and characterization of NVP-BEZ235, a new orally available dual phosphatidylinositol 3-kinase/ mammalian target of rapamycin inhibitor with potent in vivo antitumor activity. Mol Cancer Ther 2008;7: 1851-1863.

17 Ylipää A, Hunt KK, Yang J, et al. Integrative genomic characterization and a genomic staging system for gastrointestinal stromal tumors. Cancer 2011;117: 380-389.

18 Yang J, Ikezoe T, Nishioka C, et al. Long-term exposure of gastrointestinal stromal tumor cells to sunitinib induces epigenetic silencing of the PTEN gene. Int J Cancer 2012;130:595-966.

19 Ricci R, Maggiano N, Castri F, et al. Role of PTEN in gastrointestinal stromal tumor progression. Arch Pathol Lab Med 2004;128:421-425.

20 Liang YM, Li XH, Li WM, et al. Prognostic significance of PTEN, Ki-67 and CD44s expression patterns in gastrointestinal stromal tumors. World J Gastroenterol 2012;18:1664-1671.

21 Carracedo A, Pandolfi PP. The PTEN-PI3K pathway: of feedbacks and cross-talks. Oncogene 2008;27:5527-5541.

22 Candarlapaty S, Sawai A, Scaltriti M, et al. AKT inhibition relieves feedback suppression of receptor tyrosine kinase expression and activity. Cancer Cell 2011;19:58-71.

23 Miettinen M, Lasota J. Gastrointestinal stromal tumors: pathology and prognosis at different sites. Semin Diagn Pathol 2006;23:70-83. 